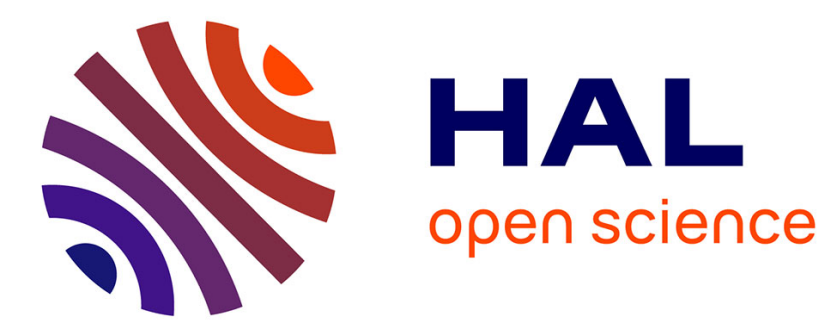

\title{
A Boundary Element Formulation for the Wave Propagation in Unsaturated soils
}

Pooneh Maghoul, Behrouz Gatmiri, Murray Fredlund, Denis Duhamel

\section{To cite this version:}

Pooneh Maghoul, Behrouz Gatmiri, Murray Fredlund, Denis Duhamel. A Boundary Element Formulation for the Wave Propagation in Unsaturated soils. 5th BIOT Conference on Poromechanics, Jul 2013, Vienna, Austria. pp.1, 10.1061/9780784412992.103 . hal-00949334

\section{HAL Id: hal-00949334 https://hal.science/hal-00949334}

Submitted on 21 Feb 2014

HAL is a multi-disciplinary open access archive for the deposit and dissemination of scientific research documents, whether they are published or not. The documents may come from teaching and research institutions in France or abroad, or from public or private research centers.
L'archive ouverte pluridisciplinaire HAL, est destinée au dépôt et à la diffusion de documents scientifiques de niveau recherche, publiés ou non, émanant des établissements d'enseignement et de recherche français ou étrangers, des laboratoires publics ou privés. 


\title{
A Boundary Element Formulation for the Wave Propagation in the Unsaturated Soils
}

\author{
Pooneh Maghoul ${ }^{1}$, Behrouz Gatmiri ${ }^{2,3}$, Murray Fredlund ${ }^{1}$, Denis Duhamel ${ }^{3}$ \\ ${ }^{1}$ SoilVision Systems Ltd., 2109 McKinnon Ave S., Saskatoon, SK S7J 1N3, Canada, \\ email: pooneh.maghoul@gmail.com; murray@soilvision.com \\ ${ }^{2}$ Department of Civil Engineering, University of Tehran, Tehran, Iran, \\ behrouz.gatmiri@andra.fr

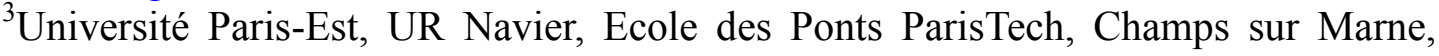 \\ France, denis.duhamel@enpc.fr
}

\begin{abstract}
This paper aims at obtaining an advanced formulation of the time-domain Boundary Element Method (BEM) for two-dimensional dynamic analysis of unsaturated soil. Unlike the usual time-domain BEM the present formulation applies a Convolution Quadrature which requires only the Laplace-domain instead of the time-domain fundamental solutions. The coupled equations governing the dynamic behavior of unsaturated soils ignoring contributions of the inertia effects of the fluids (water and air) are derived based on the poromechanics theory within the framework of the suction-based mathematical model. In this formulation, the solid skeleton displacements, water pressure and air pressure are presumed to be independent variables. As there is no analytical solution for the $2 \mathrm{D}$ wave propagation in unsaturated soils in the literature, to verify the accuracy of this implementation, the displacement response obtained by the boundary element formulation is partially verified by comparison with the elastodynamics problem.
\end{abstract}

\section{INTRODUCTION}

It has long been recognized that surface topographies can have crucial influences on damage severity and its spatial distribution during strong earthquakes. Site response analysis of topographical structures could only be solved accurately, economically and under realistic conditions, with the aid of numerical methods such as the Boundary Element Method (BEM).

Unsaturated soils are encountered near the earth's surface where most engineering structures are ultimately supported. Saturated and dry soils can become unsaturated due to seasonal variations. In the current state of the art, it could be claimed that behavior of the saturated porous media has been well understood. Conversely, the study of the dynamic behavior of unsaturated porous media is a relatively new area in the field of geotechnical earthquake engineering.

The BEM is a very effective numerical tool for dynamic analysis of linear elastic bounded and unbounded media. The method is very attractive for wave propagation problems, because the discretization is done only on the boundary, yielding smaller meshes and systems of equations. Another advantage is that this 
method represents efficiently the outgoing waves through infinite domains, which is very useful when dealing with scattered waves by topographical structures.

This paper aims at obtaining an advanced formulation of the time-domain Boundary Element Method (BEM) for two-dimensional dynamic analysis of unsaturated soil. Unlike the usual time-domain BEM the present formulation applies a Convolution Quadrature developed by Lubich (1988) which requires only the Laplace-domain instead of the time-domain fundamental solutions.

In this paper first of all, a set of fully coupled governing differential equations of a porous medium saturated by two compressible fluids (water and air) subjected to dynamic loadings is obtained. These phenomenal formulations are presented based on the experimental observations and with respect to the poromechanics theory within the framework of the suction-based mathematical model presented by Gatmiri et al. (1998). In this model, the effect of deformations on the suction distribution in the soil skeleton and the inverse effect are included in the formulation via a suctiondependent formulation of state surfaces of void ratio and degree of saturation. In this formulation, the solid skeleton displacements $u_{i}$, water pressure $p_{w}$ and air pressure $p_{a}$ are presumed to be independent variables. Secondly, the Boundary Integral Equation (BIE) is developed directly from those equations via the use of the weighted residuals method in a way that permits an easy discretization and implementation in a numerical code. The associated fundamental solution obtained by Maghoul et al. (2011) is used in the BIE.

Finally, as it seems that there is no analytical solution for the $2 \mathrm{D}$ wave propagation in unsaturated soils in the literature, to verify the accuracy of this implementation, the displacement response obtained by the boundary element formulation is partially verified by comparison with the elastodynamic problem.

\section{GOVERNING EQUATIONS}

Governing differential equations consist of mass conservation equations of liquid and gaseous phases, the equilibrium equation of the skeleton associated with water and air flow equations and constitutive relation. The assumption of infinitesimal transformation and incompressibility of solid matrix is considered.

Solid Skeleton. The equilibrium equation and the constitutive law for the soil's solid skeleton including the effect of suction are written:

$$
\begin{gathered}
\left(\sigma_{i j}-\delta_{i j} p_{a}\right)_{, j}+p_{a, i}+f_{i}=\rho \ddot{u}_{i} \\
\left(\sigma_{i j}-\delta_{i j} p_{a}\right)=\left(\lambda \delta_{i j} \varepsilon_{k k}+2 \mu \varepsilon_{i j}\right)-F_{i j}^{s}\left(p_{a}-p_{w}\right)
\end{gathered}
$$

where $\mu, \lambda$ are Lame coefficients, $p_{\alpha=w, a}$ is the water or air pressure, $\delta_{i j}$ is the Kronecker delta and $F_{i j}^{S}$ is the suction modulus matrix:

$$
F_{i j}^{S}=D_{i j k l}\left(D_{l k}^{S}\right)^{-1}
$$

in which $D_{l k}^{S}$ is a vector obtained from the state surface of void ratio $(e)$ which is a function of the independent variables of $\left(\sigma-p_{a}\right)$ and $\left(p_{a}-p_{w}\right)$ :

$$
\left(D_{k l}^{S}\right)^{-1}=\left(\partial e /\left((1+e) \partial\left(p_{a}-p_{w}\right)\right)\right)\left[\begin{array}{lll}
1 & 1 & 0
\end{array}\right]^{T}
$$


The elasticity matrix $\left(D_{i j k l}\right)$ can be presented by using the bulk modulus and the tangent modulus:

$$
D_{i j k l}(\lambda, \mu)=D_{i j k l}\left(K_{0}, E_{t}\right)=D_{i j k l}\left(\sigma-p_{a}, p_{a}-p_{w}\right)
$$

where $E_{t}$ is tangent elastic modulus which can be evaluated as:

$$
E_{t}=E_{l}+E_{s}
$$

in which $E_{l}$ is the elastic modulus in absence of suction and

$$
E_{s}=m_{s}\left(p_{a}-p_{w}\right)
$$

$m_{s}$ being a constant, $E_{s}$ represents the effect of suction on the elastic modulus. $K_{0}$ is the bulk modulus of an open system and evaluated from the surface state of void ratio:

$$
K_{0}^{-1}=(1 /(1+e)) \partial e / \partial\left(\sigma-p_{a}\right)
$$

Mass Conservation of Water. The mass of the water in a representative elementary volume can be written as:

$$
\dot{w}_{i, i}^{w}=-S_{w} \dot{\varepsilon}_{i i}+C_{w w} \dot{p}_{w}+C_{w a} \dot{p}_{a}
$$

where $C_{w w}=\left(n g_{1}-C_{w} n S_{w}\right)$ and $C_{w a}=-n g_{1}$.

In this equation, $w_{i}^{\alpha=w, a}$ is the displacement of water or air relative to solid, $S_{\alpha=w, a}$ is the degree of saturation relative to water or air, $C_{\alpha=w, a}$ is the compressibility of water or air $C_{\alpha=w, a}=d \rho_{\alpha} /\left(\rho_{\alpha} d p_{\alpha}\right)$ and $g_{1}=d S_{w} / d\left(p_{a}-p_{w}\right)$.

Mass Conservation of Air. With the same approach presented before, the mass conservation of the air can be written as:

$$
\dot{w}_{i, i}^{a}=-S_{a} \dot{\varepsilon}_{i i}+C_{a w} \dot{p}_{w}+C_{a a} \dot{p}_{a}
$$

where $C_{a a}=\left(n g_{1}-C_{a} n S_{a}\right)$ and $C_{a w}=-n g_{1}$.

Flow Equation for the Water. Based on generalized Darcy's law for describing the balance of the forces acting on the liquid phase of the representative elementary volume, the water velocity in the unsaturated soil takes the following form:

$$
-p_{w, i}=\rho_{w} \ddot{u}_{i}+\dot{w}_{i}^{w} / k_{w}-\rho_{w} g_{i}
$$

in which $k_{w}$ denotes the water permeability in an unsaturated soil.

Flow Equation for the Air. With the same approach presented for the water based on generalized Darcy's law, the air velocity in the unsaturated soil takes the following form:

$$
-p_{a, i}=\rho_{a} \ddot{u}_{i}+\dot{w}_{i}^{a} / k_{a}-\rho_{a} g_{i}
$$

in which $k_{a}$ denotes the air permeability in an unsaturated soil.

Summary of the Governing Differential Equations in Laplace Domain. By introducing (2) into (1), (11) into (9) and (12) into (10) and by applying the Laplace transform to eliminate the time variable of partial differential equations, assuming $u_{i(t=0)}=w_{i(t=0)}^{w}=w_{i(t=0)}^{a}=0$ and $p_{w(t=0)}=p_{a(t=0)}=0$, we obtain the final set of governing equations in Laplace transform domain:

$$
\mathbf{B}\left[\begin{array}{lll}
\tilde{u}_{\alpha} & \tilde{p}_{w} & \tilde{p}_{a}
\end{array}\right]^{T}+\left[\begin{array}{lll}
\tilde{f}_{\alpha} & 0 & 0
\end{array}\right]^{T}=0
$$


with the not self-adjoint operator $\mathbf{B}$ :

$$
\mathbf{B}=\left[\begin{array}{ccc}
\left(\mu \Delta-\rho s^{2}\right) \delta_{\alpha \beta}+(\lambda+\mu) \partial_{\alpha} \partial_{\beta} & F^{s} \partial_{\alpha} & \left(1-F^{s}\right) \partial_{\alpha} \\
-s \theta_{1} \partial_{\beta} & k_{w} \Delta+C_{w w} s & C_{w a} s \\
-s \theta_{2} \partial_{\beta} & C_{w a} s & k_{a} \Delta+C_{a a} s
\end{array}\right]
$$

where $\theta_{1}=\left(S_{w}-\rho_{w} k_{w} s\right)$ and $\theta_{2}=\left(S_{a}-\rho_{a} k_{a} s\right)$.

In equations (13) and (14), $\alpha, \beta=\overline{1,2}$ in two dimensional problems. Also in (14), the partial derivative ( ),, , is denoted by $\partial_{\alpha}$ and $\Delta=\partial_{\alpha \alpha}$ is the Laplacian operator.

\section{BOUNDARY INTEGRAL EQUATION}

The boundary integral equations for this problem will be derived by taking the fundamental solution as the weighted function and using the method of weighted residuals, which is essentially an integration by parts technique. In this method, the integral equation is derived directly by equating the inner product of eq. (13) and the matrix of the adjoint fundamental solutions $\widetilde{\mathbf{G}}$ implying that

$$
\mathbf{B}^{*} \widetilde{\mathbf{G}}+\mathbf{I} \delta(x-\xi)=\mathbf{0}
$$

to a null vector, i.e. $\int_{\Omega} \mathbf{B}\left[\begin{array}{ccc}\tilde{u}_{\alpha} & \tilde{p}_{w} & \tilde{p}_{a}\end{array}\right]^{T} \widetilde{\mathbf{G}} d \Omega=0$ with

$$
\widetilde{\mathbf{G}}=\left[\begin{array}{ccc}
\tilde{G}_{\alpha \beta} & \tilde{G}_{\alpha w} & \tilde{G}_{\alpha a} \\
\tilde{G}_{w \beta} & \tilde{G}_{w w} & \tilde{G}_{w a} \\
\tilde{G}_{a \beta} & \tilde{G}_{a w} & \tilde{G}_{a a}
\end{array}\right]=\left[\begin{array}{ccc}
\widetilde{U}_{\alpha \beta}^{S} & \widetilde{U}_{\alpha}^{w} & \widetilde{U}_{\alpha}^{A} \\
\tilde{P}_{\beta}^{w S} & \tilde{P}^{w W} & \tilde{P}^{w A} \\
\tilde{P}_{\beta}^{a S} & \tilde{P}^{a W} & \tilde{P}^{a A}
\end{array}\right]
$$

where the integration is performed over a domain $\Omega$ with boundary $\Gamma$ and vanishing body forces and sources are assumed. In eq. (15), $\mathbf{B}^{*}$ is the adjoint operator to $\mathbf{B}$ used for the deduction of fundamental solutions (Maghoul et al. 2011).

After integrating by parts twice over the domain according to the theory of Green's formula and using partial integration, the operator $\mathbf{B}$ is transformed from acting on the vector of unknowns $\left[\begin{array}{ccc}\tilde{u}_{\alpha} & \tilde{p}_{w} & \tilde{p}_{a}\end{array}\right]^{T}$ to the matrix of fundamental solutions $\widetilde{\mathbf{G}}$. After some algebraic manipulations and using the property of Dirac's delta function, we reach the transformed dynamic unsaturated poroelastic boundary integral representation for the transformed internal displacements and pressures given in matrix form, i.e.,

$$
\begin{aligned}
{\left[\begin{array}{ccc}
c_{\alpha \beta} & 0 & 0 \\
0 & c & 0 \\
0 & 0 & c
\end{array}\right] \mathbf{I}\left[\begin{array}{c}
\tilde{u}_{\alpha} \\
\tilde{p}_{w} \\
\tilde{p}_{a}
\end{array}\right] } & \\
& =\int_{\Gamma}\left[\begin{array}{ccc}
\widetilde{U}_{\alpha \beta}^{S} & -\tilde{P}_{\alpha}^{w S} & -\tilde{P}_{\alpha}^{a s} \\
\widetilde{U}_{\beta}^{W} & -\tilde{P}^{w W} & -\tilde{P}^{a W} \\
\widetilde{U}_{\beta}^{A} & -\tilde{P}^{w A} & -\tilde{P}^{a A}
\end{array}\right]\left[\begin{array}{c}
\tilde{t}_{\alpha} \\
\tilde{q}_{w} \\
\tilde{q}_{a}
\end{array}\right] d \Gamma \\
& -\int_{\Gamma}\left[\begin{array}{ccc}
\tilde{T}_{\alpha \beta}^{S} & \tilde{Q}_{\alpha}^{w S} & \tilde{Q}_{\alpha}^{a S} \\
\tilde{T}_{\beta}^{W} & \tilde{Q}^{w W} & \tilde{Q}^{a W} \\
\tilde{T}_{\beta}^{A} & \tilde{Q}^{w A} & \tilde{Q}^{a A}
\end{array}\right]\left[\begin{array}{c}
\tilde{u}_{\alpha} \\
\tilde{p}_{\mathrm{w}} \\
\tilde{p}_{\mathrm{a}}
\end{array}\right] d \Gamma
\end{aligned}
$$

where the traction vector, the normal water flux and the normal air flux are respectively: 


$$
\begin{gathered}
\tilde{t}_{\alpha}=\sigma_{\alpha \beta} n_{\beta}=\left[\left(\lambda \tilde{u}_{k, k}-F^{s}\left(\tilde{p}_{a}-\tilde{p}_{w}\right)+\tilde{p}_{a}\right) \delta_{\alpha \beta}+\mu\left(\tilde{u}_{\beta, \alpha}+\tilde{u}_{\alpha, \beta}\right)\right] n_{\beta} \\
\tilde{q}_{\mathrm{w}}=-k_{w}\left(\tilde{p}_{w, n}+\rho_{w} s^{2} \tilde{u}_{\alpha} n_{\alpha}\right) \\
\tilde{q}_{a}=-k_{a}\left(\tilde{p}_{a, n}+\rho_{a} s^{2} \tilde{u}_{\alpha} n_{\alpha}\right)
\end{gathered}
$$

The coefficient $c_{i j}$ has a value $\delta_{i j}$ for points inside $\Omega$ and zero outside $\Omega$. The value of $c_{i j}$ for points on the boundary $\Gamma$ is determined from the Cauchy principal value of the integrals.

The corresponding 2D fundamental solutions $\widetilde{\mathbf{G}}$ as well as the $\widetilde{\mathbf{T}}^{S^{*}}, \widetilde{\mathbf{Q}}^{w s^{*}}$ and $\widetilde{\mathbf{Q}}^{a S^{*}}$ in Eq. (17) which can be interpreted as the adjoint terms to the traction vector $\tilde{t}_{\alpha}$, the water flux $\tilde{q}_{\mathrm{w}}$ and the air flux $\tilde{q}_{\mathrm{a}}$ were already derived in Maghoul et el. (2011).

The time dependent boundary integral equation for the unsaturated soil is obtained by a transformation to time domain:

$$
c_{i j}(\xi) u_{j}(\xi ; t)=\int_{0}^{t} \int_{\Gamma}\left[G_{i j}^{*}(x, \xi ; t-\tau) t_{j}(x ; \tau)-F_{i j}^{*}(x, \xi ; t-\tau) u_{j}(x ; \tau)\right] d \Gamma
$$

\section{BOUNDARY ELEMENT FORMULATION}

Eq. (21) is an exact represent of the dynamic response of a multiphase porous medium, involving integrations over the surface as well as the time history. For the practical problem, suitable approximations are needed for both spatial and temporal variations of field variables. As will be shown, temporal integrations of the time functions involved will be performed numerically using an operational convolution quadrature method (CQM), as like as the spatial integration which be evaluated using numerical techniques. The salient features of the temporal and spatial integrations are outlined below.

Temporal integration. Since it seems too difficult to obtain the time-dependent fundamental solution in an explicit analytical form by an inverse transformation of the frequency domain results, the convolution quadrature method (CQM) proposed by Lubich (1988 a, b) is used. In this formulation, the convolution integral is numerically approximated by a quadrature formula whose weights are determined by the Laplace transform of the fundamental solution and a linear multistep method (Schanz and Antes, 1997). By applying this method, the convolution integrals between the fundamental solutions and the nodal values in eq. (21) are approximated by:

$$
\begin{aligned}
& \int_{0}^{\mathrm{t}} G_{i j}^{*}(x, \xi ; t-\tau) t_{j}(x ; \tau) d \tau=\sum_{n=1}^{N}\left(\omega_{i j}^{G}\right)^{N-n+1}(x, \xi) t_{j}^{n}(x) \\
& \int_{0}^{\mathrm{t}} F_{i j}^{*}(x, \xi ; t-\tau) u_{j}(x ; \tau) d \tau=\sum_{n=1}^{N}\left(\omega_{i j}^{F}\right)^{N-n+1}(x, \xi) u_{j}^{n}(x)
\end{aligned}
$$

in which $\left(\omega_{i j}^{G}\right)^{N-n+1}$ and $\left(\omega_{i j}^{F}\right)^{N-n+1}$ are the influence function which are defined by: 


$$
\begin{aligned}
& \left(\omega_{i j}^{G}\right)^{m}(x, \xi)=\frac{\mathcal{R}^{-m}}{L} \sum_{l=0}^{L-1} \tilde{G}_{i j}^{*}\left(x, \xi ; s_{l}\right) e^{-i m l \frac{2 \pi}{L}} \\
& \left(\omega_{i j}^{F}\right)^{m}(x, \xi)=\frac{\mathcal{R}^{-m}}{L} \sum_{l=0}^{L-1} \tilde{F}_{i j}^{*}\left(x, \xi ; s_{l}\right) e^{-i m l \frac{2 \pi}{L}}
\end{aligned}
$$

where $s_{l}$ is given by $s_{l}=\gamma\left(\mathcal{R} e^{-2 \pi i l / L}\right) / \Delta t$.

By substituting Eqs. (22) and (23) into Eq. (21), the time-convoluted boundary element equation is:

$$
\begin{aligned}
c_{i j}(\xi) u_{j}^{N}(\xi)= & \sum_{n=1}^{N}\left(\int_{\Gamma}\left(\omega_{i j}^{G}\right)^{N-n+1}(x, \xi) t_{j}^{n}(x) d \Gamma\right. \\
& \left.-\int_{\Gamma}\left(\omega_{i j}^{F}\right)^{N-n+1}(x, \xi) u_{j}^{n}(x) d \Gamma\right)
\end{aligned}
$$

Spatial integration. In this section in order to numerically evaluate the integrals on the surface $\Gamma$ (Eq. (26)), a spatial discretization will be performed. Using isoparametric quadratic elements and assuming a quadratic variation over both geometry and field variables, the functions (displacements and tractions) at any point over an element can be expressed in terms of the nodal values as $u_{i}=N_{m}(\eta) U_{i m}^{e}$, $t_{i}=N_{m}(\eta) T_{i m}^{e}, p_{w}=N_{m}(\eta) P_{w_{m}}^{e}, q_{w}=N_{m}(\eta) Q_{w m}^{e}, p_{a}=N_{m}(\eta) P_{a_{m}}^{e}$ and $q_{a}=$ $N_{m}(\eta) Q_{a_{m}}^{e}$ where $i=1,2$ for $2 \mathrm{D}$ and $m=1,2,3$ for a quadratic element and $N_{m}$ are the shape functions in the local intrinsic coordinates $(\eta)$ of the element. Once the spatial discretization process described above has been accomplished, the nodal quantities can be brought outside the surface integrals of Eq. (26), since now the integrands contain only known functions. Therefore, the discretized BE equation corresponding to point $\xi$ can be written as:

$$
\begin{aligned}
c_{i j}(\xi) u_{j}^{N}(\xi)= & \sum_{n=1}^{N} \sum_{e=1}^{E} \sum_{m=1}^{M=3}\left[\left(T_{j m}^{e}\right)^{n}\left(\Delta G_{i j_{m}}^{e}\right)^{N-n+1}\right. \\
& \left.-\left(U_{j m}^{e}\right)^{n}\left(\Delta F_{i j_{m}}^{e}\right)^{N-n+1}\right]
\end{aligned}
$$

in which the integrals which have to be evaluated over the isoparametric element $\Gamma_{e}$, can be written in intrinsic coordinates. Then:

$$
\begin{aligned}
& \left(\Delta G_{i j_{m}}^{e}\right)^{N-n+1}=\int_{-1}^{1}\left(\omega_{i j}^{G}\right)^{N-n+1}(\mathbf{x}(\eta), \xi) N_{m}(\eta)|J(\eta)| d \eta \\
& \left(\Delta F_{i j_{m}}^{e}\right)^{N-n+1}=\int_{-1}^{1}\left(\omega_{i j}^{F}\right)^{N-n+1}(\mathbf{x}(\eta), \xi) N_{m}(\eta)|J(\eta)| d \eta
\end{aligned}
$$

where $|J(\eta)|$ is the Jacobian of transformation.

The usual point collocations scheme, i.e. by allowing point $\xi$ to coincide sequentially with all the nodal points of the boundary, is used to establish a set of integral equations in order to obtain unknown boundary values. Since there are four degrees of freedom $(i=4)$ for each node of the problem $\left[u_{1}, u_{2}, p_{w}, p_{a}\right]^{T}$, there are $i=4$ integral equations per location $\xi$. Eq. (27) can be written in matrix form by replacing the double sum $\Sigma_{e=1}^{E=3} \Sigma_{m=1}^{M=3}$ by a matrix multiplication, where coefficients are assembled in a similar way as in the FEM. Then, 


$$
\overline{\mathbf{F}}^{1} \mathbf{U}^{N}=\Delta \mathbf{G}^{1} \mathbf{T}^{N}+\sum_{n=1}^{N-1}\left[\Delta \mathbf{G}^{N-n+1} \mathbf{T}^{n}-\Delta \mathbf{F}^{N-n+1} \mathbf{U}^{n}\right]
$$

\section{VERIFICATION}

As explained above, this research is the first attempt to develop BEM formulations for the problem of $2 \mathrm{D}$ wave propagation in unsaturated soils. The complexity of the coupled hydro-mechanical model in unsaturated porous media, subjected to dynamic loadings, leads to the fact that no analytical solution is known in the literature.

Therefore, the results obtained by BEM for the dynamic behavior of unsaturated soils will be partially verified by comparison with elastodynamic result which is related to a purely mechanical simulation. In this case, the nodal degrees of freedom related to water pressure and air pressure are blocked.

A $3 \mathrm{~m}$ high soil column, meshed by 32 quadratic elements on the boundary and a total of 64 nodes is considered (Fig. 1). At the upper surface of the column a sudden and constant longitudinal pressure, $t_{y}=-1 \mathrm{kN} / \mathrm{m}^{2} H(t)$ which is distributed uniformly is applied. On the side walls the movements in the horizontal direction are restricted, while at the bottom surface they are blocked in the vertical direction. In addition, all the boundaries are assumed to be permeable, i.e. the water pressure is zero. Also, the air pressure is assumed to be zero.

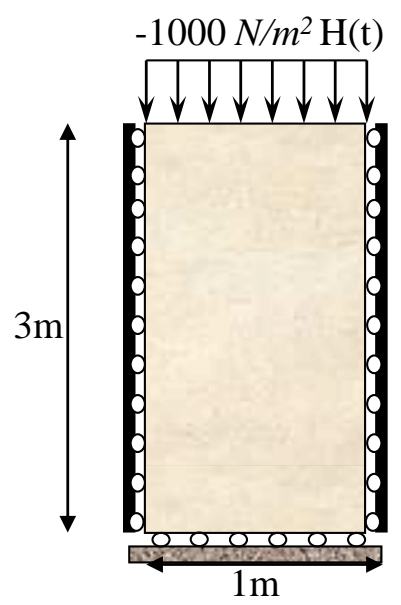

Figure 1. Unsaturated column of soil.

The hydraulic and mechanical properties of the soil are presented in the table 1 .

The result of the numerical calculation by BEM at the central point of the upper surface is compared to elastodynamic results (Fig. 2). There is a good agreement between the elastodynamic solution and the numerical results obtained by the proposed formulation. 
Table 1. Hydraulic and mechanical properties of unsaturated column

$\begin{array}{cccccc}E\left(\mathrm{~N} / \mathrm{m}^{2}\right) & K_{0}\left(\mathrm{~N} / \mathrm{m}^{2}\right) & a_{e}(-) & b_{e}(-) & e_{0}(-) & \sigma_{e}\left(\mathrm{~N} / \mathrm{m}^{2}\right) \\ 2.54 \times 10^{10} & 2.1 \times 10^{10} & 1.0 & 0.15 & 0.73 & 8.5 \times 10^{8} \\ a_{w}(\mathrm{~m} / \mathrm{s}) & \alpha_{w}(-) & S_{r u}(-) & b_{a}\left(\mathrm{~m}^{2}\right) & \mu_{a}\left(\mathrm{Ns} / \mathrm{m}^{2}\right) & a_{s}\left(\mathrm{~m}^{2} / \mathrm{N}\right) \\ 1.2 \times 10^{-8} & 5.0 & 0.05 & 1.0 \times 10^{-8} & 1.846 \times 10^{-5} & -5.0 \times 10^{-7}\end{array}$

\section{CONCLUSION}

In this paper, an advanced formulation of the time-domain Boundary Element Method (BEM) for two-dimensional dynamic analysis of unsaturated soil is obtained. Unlike the usual time-domain BEM the present formulation applies a Convolution Quadrature which requires only the Laplace-domain instead of the time-domain fundamental solutions.

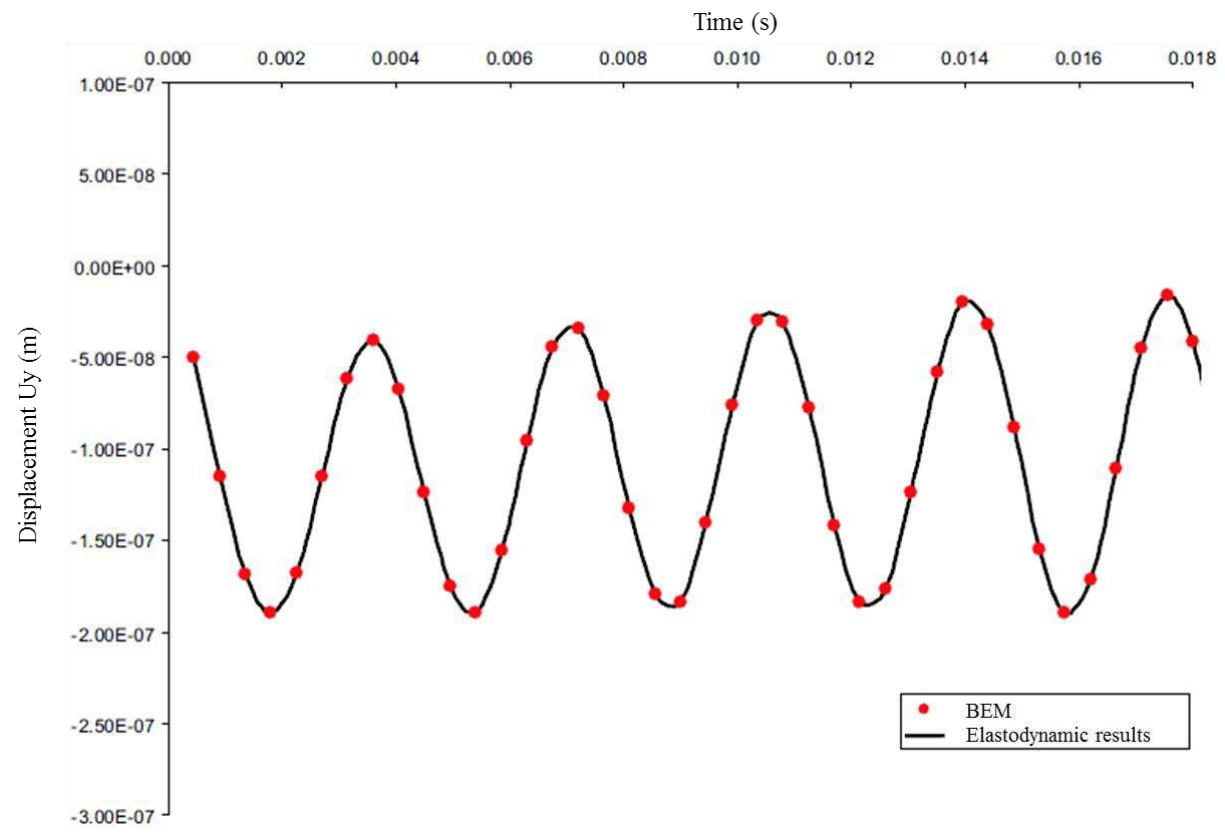

Figure 2. Vertical displacements obtained at the central point at the upper surface

\section{REFERENCES}

Gatmiri, B., Delage, P., Cerrolaza, M. (1998). "UDAM: a powerful finite element software for the analysis of unsaturated porous media." Advances in Engineering Software, 29(1), 29-43.

Lubich, C. (1988a). "Convolution quadrature and discretized operational calculus, I." Numerische Mathematik, 52, 129-145.

Lubich, C. (1988b). "Convolution quadrature and discretized operational calculus, II." Numerische Mathematik, 52, 413-425. 
Maghoul, P., Gatmiri, B., Duhamel, D. (2011). "Boundary integral formulation and two-dimensional fundamental solutions for dynamic behaviour analysis of unsaturated soils." International Journal of Soil Dynamics and Earthquake Engineering, 31(11), 1480-1495.

Schanz, M. and Antes, H. (1997). "A new visco- and elastodynamic time domain boundary element formulation.” Computational Mechanics, 20(5), 452-459. 